\title{
Der Sonderweg des Westens
}

\section{Erich Weede}

Forschungsinstitut für Soziologie, Lindenburger Allee 15, D-5000 Köln 41

Zusammenfassung: Es geht um die Frage, warum der Westen und nicht etwa die asiatischen Hochkulturen zuerst Hunger und Massenarmut durch wirtschaftliche Entwicklung überwinden konnte. Im Anschluß an Eric Jones werden geographische und geopolitische Variablen als Hintergrundbedingungen der europäischen Entwicklung analysiert. Intervenierende Variablen zwischen der Geographie und der Wirtschaftsleistung sind vor allem die Existenz eines internationalen Systems in Europa, die frühe Herausbildung einer interregionalen Arbeitsteilung und des Fernhandels mit Massengütern, relativ sichere Eigentumsrechte und aus der politischen Fragmentierung resultierende Innovationschancen. Außerdem haben vor allem die (relative) Autonomie (einiger) westlicher Städte und der westlichen Wissenschaft zur Dynamik der westlichen Entwicklung beigetragen.

\section{Einleitung}

Über die Jahrtausende waren Hunger, Elend und Unterdrückung das typische Schicksal der Menschen. Zwar gab es immer herrschende Klassen, die Überflu $\beta$ und Luxus und ein komfortables Leben genossen, aber die beherrschten Bevölkerungsmassen vegetierten am Rande des Existenzminimums. In großen Teilen Afrikas, Südasiens und sogar Lateinamerikas gilt das noch heute, in weiten Teilen Ostasiens galt es bis vor wenigen Jahrzehnten. Damit stellt sich die Frage, warum zunächst nur der Westen ${ }^{1}$ es geschafft hat, die Massenarmut zu überwinden. Vor einem halben Jahrtausend gab es ja kaum gute Gründe, die abendländische Zivilisation für irgendwie höher stehend als die asiatischen Hochkulturen zu halten. Aber nur der Westen hat sich autonom entwickelt.

Im Gegensatz zu den Dependenz- und Weltsystemtheoretikern (z. B. Bornschier 1980, Emmanuel 1972, Frank 1978a, 1978b, Galtung 1972,

${ }^{1}$ In diesem Aufsatz werde ich die Begriffe ,Westen', ,Westeuropa' und ,Europa' weitgehend synonym verwenden. ,Westeuropa' ist der Teil der geographischen Einheit Europa, der vor der Reformation katholisch und nicht etwa griechisch- oder russisch-orthodox geprägt war. ,Europa' wird hier als Abkürzung für das so verstandene ,Westeuropa' verwendet. Ohne damit hier schon kausale Behauptungen verbinden zu wollen, kann man feststellen, da $B$ sich der Kapitalismus in Westeuropa entwickelt hat und erst sehr spät und nur für eine kurze Zeit auf Rußland übergegriffen hat. Die USA und Kanada können als westeuropäische Tochtergesellschaften gelten, in denen sich der Kapitalismus ebenfalls früh, kräftig und dauerhaft entwickelt hat. ,Westeuropa' und seine Tochtergesellschaften zusammen bilden den ,Westen".
Wallerstein 1974, 1979; kritisch: Brunner 1985, Weede 1985a, 1985b) glaube ich nicht, daß das Fortdauern von Armut und Unterentwicklung in der Dritten Welt oder die Ausbeutung der Dritten Welt durch den Westen das entscheidende theoretische Problem bzw. der Schlüssel zur Erklärung für den Aufstieg des Westens ist. Natürlich hat es auch Ausbeutung gegeben. Aber die Ausbeutung eroberter Gebiete und unterworfener Völker ist offensichtlich älter als die westliche Zivilisation. Westliche und nicht-westliche Völker haben gleichermaßen Eroberung und Ausbeutung praktiziert, wo sie Gelegenheit dazu fanden. Außerhalb des Westens hat Ausbeutung und Plünderung aber nicht die Massenarmut auch nur auf Seiten der Eroberer nachhaltig beseitigt. Schon deshalb muß derAufstieg des Westens anders als durch Ausbeutung erklärt werden.

Außerdem setzt Ausbeutung militärische Überlegenheit voraus. Wer den Aufstieg des Westens wesentlich auch durch Ausbeutung erklären will (z. B. Frank 1978a, 1978b), der verwandelt im Grunde nur die eine Frage in eine andere. Dann geht es nicht mehr um die Erklärung des wirtschaftlichen Aufstiegs des Westens, sondern um die Erklärung der militärischen Überlegenheit oft relativ kleiner westlicher Streitkräfte über zuweilen wesentlich größere Armeen, ob bei der Eroberung Mexikos oder Indiens.

\section{Das europäische Wunder: Vom Konflikt über die Freiheit zum Wohlstand}

M. E. enthält das Werk 'The European Miracle' von Eric Jones (1981) den besten Ausgangspunkt für die Erklärung des Aufstiegs des Westens. Nach seiner Auffassung waren noch vor 500 Jahren die 
chinesische und die indische Zivilisation der abendländischen zumindest ebenbürtig, wenn nicht gar überlegen. Wir müssen uns deshalb fragen, warum das eher rückständige Europa die asiatischen Hochkulturen jemals unterwerfen und überholen konnte. Nach Jones (1981) sind die Unterschiede zwischen europäischen und asiatischen Landschaften eine wichtige Hintergrundbedingung unterschiedlicher wirtschaftlicher und politischer Entwicklungen.

Während es in Asien riesige homogene Gebiete gibt, wie die Ebenen des Ganges oder des Gelben Flusses, unterscheiden sich im eher kleinflächigen Westeuropa Bodenqualität, Regenfall und sonstige Witterungsbedingungen ziemlich deutlich in relativ dicht beieinander liegenden Gebieten. Im Unterschied zu den großen Kerngebieten für die Staatenbildung in Asien sind die westeuropäischen Kerngebiete relativ klein, wie beispielsweise die Ile de France. In Westeuropa haben schwieriges Gelände, Berge und Wälder die Kerngebiete trotz relativ kurzer Distanzen voneinander getrennt. Deshalb konnten chinesische und zeitweilig auch indische Herrscher riesige Gebiete vereinigen, unterwerfen und befrieden, während europäische Fürsten ziemlich kleine Gebiete beherrschten.

In der indischen und mehr noch in der chinesischen Geschichte gab es Zeiten, in denen hochzivilisierte Imperien nur relativ rückständige Völkerschaften als Nachbarn hatten. Zwar haben diese Völkerschaften manchmal Indien oder China erobert, aber dann wurden die Eroberer zur herrschenden Klasse und übernahmen die überlegene Zivilisation der Beherrschten. Im Gegensatz dazu mußten die europäischen Fürsten immer mit Nachbarstaaten rechnen, die wirtschaftlich, technologisch und militärisch zumindest annähernd gleichwertig waren. Im Gegensatz zur indischen und chinesischen Geschichte gibt es in Europa seit dem Mittelalter ein internationales System rivalisierender Staaten.

Nach Jones (1981) - ähnlich argumentieren auch Chirot (1986), Hall (1986) und Rosenberg and Birdzell (1986) - ist die politische Zersplitterung Europas wesentlich für den Aufstieg Europas verantwortlich, während sich das politisch geeinte China langsamer entwickelte. Im europäischen Staatensystem konnten lokale Herrscher nur auf ihr eigenes Land beschränkten Schaden anrichten. Zwar gab es in Europa wie in Asien Aberglauben und Ineffizienz in den herrschenden Klassen, aber die europäische Zivilisation im ganzen wurde dadurch nicht wesentlich behindert. Im Gegensatz dazu konnten unkluge politische Entscheidungen in China die Wachstumsaussichten der gesamten chinesischen Zivilisation beschränken. Dazu ein Beispiel: Im 15. Jahrhundert haben chinesische Seefahrer die Meere zwischen Ostafrika und Nordostsibirien erkundet. Aber denn haben zentrale politische Fehlentscheidungen verhindert, da $B$ es zu einem chinesischen Zeitalter der großen Entdeckungen kam.

Jones (1981: 67) faßt das so zusammen: "The record of Chinese exploration which was halted in 1430 and prevented by fiat from resumption in 1480 shows what could happen in a centralised empire that could not happen, or be enforced, in a decentralised system of states like Europe. Progress might be sluggish and frustrating but it was less likely to be permanently baulked; to pursue the example, Columbus did eventually find a sponsor. The other large societies of Eurasia that might potentially have developed as Europe did develop, tended to suffer from various disabilities including political centralism and whimsicality. Their earlier and perhaps greater intellectual promise was always stultified."

Das ist m.E. ein ganz zentraler Punkt in Jones' (1981) Erklärungsskizze, den ich selbst noch schärfer fassen möchte. Das europäische Staatensystem hat die Macht der Regierungen über die Völker sogar im Zeitalter des Absolutismus beschränkt. Europäer wie Asiaten litten zeitweise unter willkürlicher, abergläubischer und inkompetenter Herrschaft. Aber die Rivalität und Feindseligkeit unter den europäischen Fürsten und Regierungen, die oft nur kleine Teile Europas beherrschten, und die oft relativ nahen Grenzen zu Nachbarstaaten machten es einfach unmöglich, Innovation von oben zu unterbinden. Seit der Reformation besa $\beta$ noch nicht einmal die katholische Kirche einen ganz Westeuropa erfassenden Einfluß. Weder politische noch hierokratische Herrschaft konnte in Europa allzu viel Schaden anrichten.

Die Rivalität zwischen den europäischen Staaten hat zu Kriegen und in deren Gefolge zu Verwüstungen und Verelendung in Teilen Europas geführt. Aber der fast permanente Kriegszustand in Europa, d.h. die dauernde Sorge um die Sicherheit des Territoriums und die Finanzierung der Streitkräfte, hat die europäischen Herrscher auch gezwungen, sich mehr als die Herrscher indischer oder chinesischer Reiche für die wirtschaftliche Entwicklung ihres Landes, die Wohlfahrt und sogar Loyalität der Untertanen zu interessieren. Ein reicheres Land bedeutet mehr Steuerkraft und 
kann eine größere Armee unterhalten. Eine loyale Bevölkerung ist in Kriegszeiten vorteilhaft.

Flüchtlinge vor religiöser und politischer Verfolgung anderswo willkommen zu heißen, jedenfalls wenn sie neue Kenntnisse und Fertigkeiten ins Land brachten und damit den wirtschaftlichen Fortschritt beschleunigen halfen, war im Interesse der herrschenden Fürsten. Potentiell oder tatsächlich kriegführende Herrscher mußten ein Interesse an wirtschaftlicher und militärischer Innovation haben. Kriege und Flüchtlingsströme haben dafür gesorgt, daß sich Neuerungen in ganz Europa verbreiteten.

Natürlich haben die Kriege in Europa viel Leid und Zerstörung bewirkt, aber die dauernde Kriegsgefahr zwischen den Staaten hat auch das Machtgleichgewicht innerhalb der europäischen Gesellschaften zugunsten der unteren Volksschichten verändert (vgl. Andreski 1968a). Sogar heute noch läßt sich zeigen, daß Bedrohungen der nationalen Sicherheit und daraus resultierende hohe militärische Partizipationsgrade (nicht aber hohe Verteidigungsausgaben) $\mathrm{zu}$ beschleunigtem Wachstum, einer gewissen Angleichung der Einkommen und einer Verbesserung der physischen Lebensqualität beitragen (Dixon and Moon 1986, Garnier and Hazelrigg 1977, Jagodzinski und Weede 1980, Kahn 1979: 334 und 457, Kriesberg 1979: 379, Weede and Jagodzinski 1981, Weede 1983). Herrscher behandeln ihre Untertanen besser, wo oder wenn sie diese gegen auswärtige Feinde benötigen. Anderswo können sich die herrschenden Klassen der Untertanenausbeutung widmen.

Die Gestalt europäischer Landschaften hat aber nicht nur zu einem System rivalisierender Staaten geführt und damit den Schaden begrenzt, den einzelne Regierungen in Europa anrichten konnten. Gleichzeitig hat die Vielfalt europäischer Bodenqualitäten, Witterungs- und Anbaubedingungen dazu beigetragen, daß die komparativen Kostenvorteile verschiedener Gegenden bei der Produktion verschiedener Güter und damit die potentiellen Wohlfahrtsgewinne bei Spezialisierung und Handel viel offensichtlicher als in den relativ homogenen, großräumigen Flußtälern Süd- und Ostasiens sind bzw. waren. Deshalb ist es gerade in Europa früh zum Handel mit Massengütern für die Normalverbraucher und zur Entstehung von Märkten gekommen. Die innereuropäischen Unterschiede in der Landschaft und damit in den Produktionsmöglichkeiten haben die frühzeitige interregionale Arbeitsteilung angeregt.
Auf Durkheim (1977) gestützt könnte man gegen die Erklärung von (interregionaler) Arbeitsteilung und Austausch durch die günstigen Gelegenheiten und die potentiellen Vorteile dabei argumentieren. Ich würde diesem Einwand allerdings schon deshalb nicht allzu viel Gewicht beimessen, weil auch die erklärenden Variablen für Arbeitsteilung in Durkheims eigenem theoretischen Ansatz, nämlich Verdichtung und Volumen von Gesellschaften, notwendigerweise die Vorteilhaftigkeit von Arbeitsteilung und Austausch erhöhen, weil nämlich sowohl zunehmende Verdichtung als auch zunehmendes Volumen der Gesellschaft die Suche nach Tauschpartnern leichter werden läßt und damit einen Beitrag zur Senkung der Transaktionskosten leistet. M. E. resultiert aber die frühere Entwicklung einer interregionalen Arbeitsteilung und des Handels mit Gütern für den Massenkonsum nicht etwa daraus, daß Europa früher als Indien oder China eine stärker verdichtete oder voluminösere Gesellschaft war, sondern aus den von Jones (1981) angesprochenen landschaftlichen und klimatischen Unterschieden innerhalb Europas. Zunehmende Verdichtung und zunehmendes Volumen europäischer Gesellschaften und daraus resultierende Arbeitsteilung und Handel können nur erklären, warum in Europa trotz zunehmender Bodenverknappung der Lebensstandard nicht gesunken ist (vgl. North and Thomas 1973).

In Europa hat sich relativ früh ein Fernhandel mit Massengütern herausgebildet. Aber Asien kennt den Handel mit Luxusgütern schon länger als Europa. Neben der Größe der Herrschaftsgebiete ist der Unterschied zwischen Massen- und Luxusgütern deshalb so wichtig, weil er die relativ größere und frühere Sicherheit der Eigentums- und Verfügungsrechte in Europa beeinflußt hat. Denn die Herrscher sind je nach der Größe des von ihnen beherrschten Gebietes und des Wertes der Waren umherziehender Kaufleute recht unterschiedlichen Anreizen zur Konfiskation oder zum Beuteerwerb ausgesetzt. Wenn Kaufleute relativ billige Güter für den Massenkonsum (wie Salz, Holz, Getreide oder Gebrauchsweine) transportieren und relativ oft in kurzer Zeit dasselbe Gebiet durchqueren möchten, wenn die herrschenden Fürsten nur kleinflächige Gebiete regieren, dann werden rationale Herrscher nicht die Güter beschlagnahmen.

Der kleine Fürst erreicht durch Konfiskation zwar sofort einen gewissen Vorteil, aber sein Beuteerwerb und kurzfristiger Vorteil vertreibt gleichzeitig die Kaufleute aus seinem Gebiet - kleine Herrschaftsgebiete lassen sich umgehen - und vermin- 
dert damit die Chancen künftigen Beuteerwerbs. Der relativ geringe Wert der europäischen Massengüter hat es den Herrschern erleichtert, auf kurzfristigen Beuteerwerb zu verzichten und die Eigentumsrechte der umherziehenden Kaufleute zu respektieren. Der Konkurrenzkampf zwischen den Herrschern relativ kleiner Gebiete hat sie zu Zugeständnissen gegenüber den Kaufleuten gezwungen. Mit den Worten von Hall (1986: 102): "In the West, the absence of an empire removed the crucial bureaucratic block on the development of market forces; merchants persecuted in one place could always go with their capital elsewhere." Ähnliche Formulierungen findet man auch bei Weber (1981: 288) oder Collins (1980). Wer früher als andere gegen einigermaßen feste und vorhersehbare Abgabenpflichten Schutz und Sicherheit der Verfügungsrechte versprechen konnte, der lockte Handel und (einen zunächst sehr bescheidenen) Wohlstand in sein eigenes Herrschaftsgebiet.

Indem sie relativ sichere Eigentumsrechte bzw. Verfügungsrechte zugestanden haben, haben die vielen kleinen und mittelgroßen europäischen Herrscher eine gewisse Begrenzung willkürlicher Regierungsmacht hingenommen. Die Herrscher indischer oder chinesischer Großreiche waren nie vergleichbaren $Z$ wängen ausgesetzt. Außerdem ist es schwer, der Versuchung der Beschlagnahmung zu widerstehen, wenn wertvolle Luxuswaren das eigene Gebiet durchqueren. Der Unterschied in der Entwicklung europäischer und asiatischer Eigentums- und Verfügungsrechte ist also aus der unterschiedlichen Situation erklärbar und durchaus mit der Auffassung kompatibel, da $\beta$ europäische Herrscher genauso raffgierig wie asiatische und genauso wenig altruistisch wie jene waren.

Das Verhältnis zwischen der Stärke von Staat und Regierung einerseits und der Sicherheit der Eigentums- und Verfügungsrechte andererseits ist immer eine heikle Angelegenheit. Um der anarchistischen Ungewißheit und Unsicherheit aller Eigentums- und Verfügungsrechte $\mathrm{zu}$ entkommen, ist ein Staatsapparat notwendig (Tullock 1974). Aber wer schützt das Volk, d.h. vor allem auch die produktiv Tätigen, vor den Garanten der Eigentumsrechte und deren am Beuteerwerb orientierten Neigungen? ${ }^{2}$ In Europa sind diese Neigungen der herrschenden Klassen durch die zwischenstaatliche Rivalität und das daraus resultierende Interesse der Regierungen am Wohlergehen und der Loyalität der Untertanen, die später zu Staatsbürgern wurden, begrenzt worden. Man kann in der Entstehung und Entwicklung sicherer Eigentums- und Verfügungsrechte die zentrale Determinante sehen, die das europäische Wunder ermöglicht hat (Jones 1981, North and Thomas 1973, North 1981, Ekelund and Tollison 1981). Wenn es Produktionsanreize geben soll und Anreize zum Sparen und Investieren - und von diesen Anreizen hängen wirtschaftliche Entwicklung und Wachstum ab dann müssen die Eigentums- und Verfügungsrechte einigermaßen klar und sicher sein, dann darf der Staat keine Ausbeutungs- und Plünderungsmaschine sein.

Der Staat muß also einerseits stark genug sein, Eigentums- und Verfügungsrechte durchzusetzen; er darf andererseits nicht so stark sein, daß die Inhaber der Staatsgewalt alles, was ihnen in den Sinn kommt, durchsetzen können. Heutzutage denkt man bei der Aufgabe der Begrenzung politischer Macht zuerst und vor allem an konstitutionelle Mittel. In der Geschichte Europas ergab sich die Begrenzung aber zunächst und vor allem aus der Existenz eines internationalen Systems rivalisierender Staaten, das in der chinesischen und indischen Geschichte eher kurzfristige Ausnahme als langfristige Regel war. Man kann m. E. sogar heute noch die Frage aufwerfen, ob verfassungsmäßige Begrenzungen der Regierungsmacht allein noch ausreichen würden, wenn die internationale Rivalität zwischen den Staaten jemals für längere Zeiträume überwunden werden könnte (ähnlich: Chirot 1986: 296).

Kleinflächigkeit und Heterogenität der europäischen Landschaften haben nicht nur a) über das System rivalisierender Staaten Innovation und Innovationsdiffusion durch Flüchtlinge ermöglicht und zur Disziplinierung der Herrschenden beigetragen, sie haben nicht nur b) über regionale Arbeitsteilung und den Fernhandel mit Massengütern die Entstehung relativ sicherer Eigentumsrechte begünstigt, sondern sie haben c) auch noch bei Katastrophen und Krieg die Zerstörung der Kapi-

${ }^{2}$ Vor kurzem hat Tilly (1985) auf die Ähnlichkeiten zwischen (kriegerischen) Staatsgründungen und organisiertem Verbrechen hingewiesen. Andreski (1968b: 92) hat sogar den Begriff der Kleptokratie geprägt, um den Charakter durchaus nicht seltener Herrschaftssysteme zu beschreiben. - - Wer sich an ahistorischen theoretischen Überlegungen und provozierenden (aber $\mathrm{m}$. E. treffenden) Formulierungen stört, sollte sich vergegenwärtigen, daß Nomaden, Bergstämme und vor allem Steppenvölker immer wieder zivilisiertere und produktivere Gesellschaften erobert und beherrscht, ausgeplündert und versklavt haben (Rüstow 1950, McNeill 1982, Hall 1986). 
talbestände in Grenzen gehalten. ${ }^{3}$ In Asien bestand das Kapital vielfach aus verwundbaren Dämmen und Bewässerungsanlagen, deren Zerstörung die Fruchtbarkeit großer Flächen für lange Zeit beeinträchtigen konnte. Die europäischen Kapitalgüter waren häufiger atomistisch, wie gerodetes Land, Hecken, Wege, Häuser, Vieh, Vorräte und kleine Werkstätten. Zerstörungen bei Kriegen waren zwar in Europa häufig, aber die Auswirkungen eher kleinflächig als großflächig. Weil europäische Kriege und Katastrophen häufig mehr Menschen als Kapital vernichteten, waren hinterher die wirtschaftlichen Erholungschancen besser als in Asien, wo Mensch und Kapital meist gleichermaßen dezimiert wurden.

Die relativ sicheren Eigentumsrechte in Europa haben stärkere Investitionsanreize als in Asien vermittelt. Zusammen mit dem relativ geringen Aus$\mathrm{ma} \beta$ der Kapitalzerstörung in Kriegen und bei anderen Katastrophen hat das zu einem wachsenden Kapitalbestand in Europa geführt, der, weil immer mehr Kapital pro Arbeitskraft zur Verfügung stand, zur steigenden Produktivität der Arbeit und damit zum europäischen Wunder beitragen konnte.

Der Gegensatz zwischen einem stabilen Imperium einerseits und politischer Zersplitterung andererseits differenziert vor allem zwischen China, das vor ca. 500 Jahren Europa noch technisch überlegen war, und Europa. In Indien und im islamischen Kulturkreis gab es über Jahrhunderte auch keine stabilen und alles umfassenden Großreiche. Aber dort hat sich im Gegensatz zum mittelalterlichen Europa kein autonomes Stadtbürgertum entwickelt, worauf Max Weber $(1978,1981)$ schon früh und mit Nachdruck hingewiesen hat. Dieser Gedankengang ist in neuerer Zeit vor allem von Collins (1980) und Chirot (1986) aufgegriffen und weiterverfolgt worden. In Europa haben die feudalistische Machtteilung zwischen Oberherren und Vasallen und damit verbunden die Machtkämpfe zwischen ihnen den Städten Spielraum verschafft. Die Städte waren zwar militärisch schwächer als die lokalen und überregionalen Herren im Mittelalter, aber ihre Steuerkraft machte sie dennoch zu umworbenen Verbündeten.

\footnotetext{
${ }^{3}$ Chirot (1986) weist auch zurecht darauf hin, daB Westeuropa weiter als China, Nordindien oder die islamischen Kulturzentren von der eurasischen Steppe entfernt ist, daß auch die mittel- und osteuropäischen Wälder das Vordringen der berittenen Steppekrieger zumindest erschweren mußten.
}

Relativ autonom konnten die europäischen Städte nur werden, weil sie mehr waren als Wohnorte von Handwerkern und Kaufleuten oder Marktplätze. Weber (1981, S. 275/276) hebt folgende Merkmale hervor: „Die okzidentale Stadt in ihren Anfängen ist zunächst Wehrverband, Vereinigung der ökonomisch Wehrhaften, die imstande sind, sich selbst auszurüsten und ausbilden zu lassen ... Religiöse Verbrüderung und militärische Selbstequipierung haben die Entstehung und Existenz der Stadt ermöglicht." Die bewaffneten Mitglieder des Wehrverbandes Stadt aber habe nicht nur Pflichten, sondern auch Rechte. Zumindest unter den bewaffneten Männern gibt es deshalb auch egalitärdemokratische Tendenzen. Diesen Gedanken hat später Andreski (1968a) weiterverfolgt, der einen Zusammenhang zwischen dem militärischen Partizipationsgrad und der Gleichheit der Privilegien oder Einkommen in Gesellschaften behauptet.

Die Stadt als weitgehend autonomer Wehrverband ist keine Selbstverständlichkeit, sondern eine Ausnahme. Im politisch geeinten China ließen die Herrscher derartige Entwicklungen nicht aufkommen. Im islamischen Kulturkreis spielten neben einigen Großreichen vor allem Stammeskrieger aus Wüsten, Steppen und Bergen eine entscheidende Rolle (Hall 1986). In Indien haben die politischen Implikationen der Religion städtische Verbrüderung und Wehrhaftigkeit und damit autonome Städte verhindert. Das Kastenwesen errichtet ja rituelle Schranken zwischen den Kasten, die soweit gehen, daß selbst gemeinsame Mahlzeiten ausgeschlossen werden. Außerdem ist die Kriegführung Aufgabe der Kriegerkasten, der Handel aber anderen Kasten zugewiesen. Die heterodoxen Abspaltungen vom Hinduismus, d. h. vor allem der Buddhismus und der Jainismus, die vor allem auch unter den Städtern viele Anhänger rekrutierten, haben mit ihrem absoluten Tötungsverbot vollends wehrhafte Städte unmöglich gemacht (Weber 1978, vgl. auch Hall 1986).

Der Konflikt zwischen Oberherren und Vasallen und nach Autonomie strebenden Städten wurde im mittelalterlichen Europa durch den Konflikt zwischen Kaiser und Papst weiter kompliziert. Das Ergebnis dieser Auseinandersetzungen war der Ständestaat mit spezifischen Privilegierungen und Mitspracherechten der Städte in Landtagen, Generalständen und Parlamenten - vor allem auch bei der Besteuerung. In England und in den Niederlanden haben sich diese mittelalterlichen Formen der Gewaltenteilung länger als in absolutistisch regierten Regionen gehalten. Genau dort, in Hol- 
land und England ${ }^{4}$, hat dann die Kommerzialisierung und das Wirtschaftswachstum auch früher und nachhaltiger als anderswo in Europa eingesetzt (Chirot 1986).

Schon im Mittelalter wurden die europäischen Städte Zentren der Kommerzialisierung. Sie haben auch die spezifisch europäische Rechtsentwicklung und das europäische Religionsverständnis mitgeprägt. Wirtschaft und Handel benötigen berechenbare Rahmenbedingungen, Vorhersehbarkeit, Kalkulierbarkeit - also ein rationales, herrschaftlicher Willkür entzogenes, professionell angewandtes Recht. Mit den Worten Max Webers (1981: 240): „Der kapitalistische Wirtschaftsbetrieb muß sich, wenn er rational wirtschaften soll, darauf verlassen können, da $\beta$ berechenbar judiziert und verwaltet wird. Weder in der Zeit der hellenischen Polis, noch in den Patrimonialstaaten Asiens, noch in den abendländischen bis zu den Stuarts war dies gewährleistet. Die königliche Billigkeitsjustiz mit ihren Gnadenerlassen brachte fortwährend Störungen in die Berechnungen des Wirtschaftslebens." 5

Notwendige Hintergrundbedingung eines berechenbaren Rechts ist die Einschränkung der Herrschaft oder ihre ,Domestikation“ (Albert 1986). Gerade weil es im mittelalterlichen Europa weder innerhalb der entstehenden Staaten noch zwischen den Staaten auf Dauer eindeutige Sieger gab, konnte sich ein kalkulierbares Recht entwickeln. Chirot (1986: 23) faßt das so zusammen: "The main reason for legal rationalization in the West,

${ }^{4} \mathrm{Da}$ die britischen Inseln bei der Kommerzialisierung und frühkapitalistischen Entwicklung Europas eine so wichtige Rolle spielten, widerspricht $m$. E. nicht einem Erklärungsansatz, der vor allem auch (aber nicht nur!) die Existenz eines zwischenstaatlichen Systems und die zwischenstaatliche Rivalität für den Sonderweg des Westens verantwortlich macht. Denn England war sowohl sicherheitspolitisch als auch kulturell als auch wirtschaftlich Teil des europäischen Staatensystems. Kapital und Talent konnten England nicht schwerer als andere europäische Staaten verlassen oder erreichen. Die sicherheitspolitisch privilegierte Randlage im europäischen Staatensystem hat dazu beigetragen, $\mathrm{da} B$ das Land nie so große Landstreitkräfte wie vergleichbare große Kontinentalstaaten unterhalten mußte, daß der König nie soviel Macht wie einige absolute Herrscher auf dem Kontinent durchsetzen konnte. Der Fall England erinnert allerdings daran, daß es neben der Einschränkung politischer Macht durch zwischenstaatliche Rivalität auch noch eine Einschränkung politischer Macht durch innerstaatliche Gleichgewichte (z. B. zwischen König und Adel) geben kann. then, was the long, indecisive multisided political struggle between kings, nobles, the church and the towns. The emergence of a kind of working truce institutionalized by the Ständestaat created the conditions for stable, rational legal rule which enhanced the possibility of rational economic behavior." Oder man könnte dasselbe mit Albert (1986: 21) etwas allgemeiner fassen und herausarbeiten, „daß die Entstehung von Spielräumen für autonome Kräfte und damit von Freiheit nicht selten das Resultat von Konflikten ist, in denen es keiner der beteiligten Mächte gelingt, die Oberhand zu gewinnen."

Relativ sichere Eigentumsrechte oder relativ geringe Konfiskationsgefahr, Stadt- und Bürgerrechte, eine faktische Gewaltenteilung haben in Europa Herrschaft und Willkür begrenzt und ein rationales Recht entstehen lassen. Im Zeitalter des Absolutismus verloren die meisten europäischen Städte ihre Freiheiten. Wie konnten dennoch die Ansätze zu einer berechenbaren, wirtschaftsfreundlichen Rechtsentwicklung fortentwickelt werden? Weber (1981: 288/289) verweist darauf, daß die Städte ,in die Gewalt konkurrierender Nationalstaaten (gerieten), die in ständigem friedlichen und kriegerischen Kampf um die Macht lagen. Dieser Konkurrenzkampf schuf dem neuzeitlich-abendländischen Kapitalismus die größten Chancen. Der einzelne Staat mußte um das freizügige Kapital konkurrieren, das ihm die Bedingungen vorschrieb, unter denen es ihm zur Macht verhelfen wollte. Aus dem notgedrungenen Bündnis des Staates mit dem Kapital ging der nationale Bürgerstand hervor, die Bourgeoise im modernen Sinn des Wortes. Der

${ }^{5}$ Die Vorhersehbarkeit der Rechtssprechung ist allerdings nicht allein entscheidend. Auch auf die inhaltliche Ausgestaltung des Rechts kommt es an. Sofern das positive Recht restriktive Praktiken und Monopole schützt, kann auch die Unmöglichkeit derartiges Recht durchzusetzen zur wirtschaftlichen Entwicklung beitragen. Nach Ekelund and Tollison (1981) haben sich England und Frankreich zur Zeit des Merkantilismus dadurch unterschieden, daß im absolutistischen Frankreich Privilegien und restriktive Praktiken seitens der königlichen Verwaltung und Justiz durchgesetzt werden konnten, daß das in England mit seiner Rivalität von König und Parlament, von königlichen und common law Gerichten aber nicht möglich war. Wo Monopole und Privilegien kaum durchsetzbar sind, da bemühen sich dann rationale Akteure auch weniger darum. Nach Ekelund and Tollison (1981) ist das ein entscheidender Grund dafür, daß sich England besser bzw. früher als Frankreich entwickelt hat. 
geschlossene nationale Staat also ist es, der dem Kapitalismus die Chance des Fortbestehens gewährleistet; solange er nicht einem Weltreich Platz macht, wird also auch der Kapitalismus dauern." Auch im Zeitalter des Absolutismus hat die zwischenstaatliche Rivalität die Herrschenden zur Rücksicht auf die Wirtschaft gezwungen.

Die geopolitische Zersplitterung Europas hat im wesentlichen die Dezentralisierung politischer Macht im abendländischen Kulturkreis bewirkt. Mit Rosenberg and Birdzell (1986) kann man auBerdem auch die später erfolgende Trennung politischer von wirtschaftlicher Macht für den europäischen Sonderweg verantwortlich machen. Im Mittelalter lagen politische und wirtschaftliche Macht im wesentlichen in denselben Händen. Die überwältigende Mehrheit der Bevölkerung lebte auf dem Lande und arbeitete in der Landwirtschaft. Guts- und Feudalherren boten militärischen Schutz und sprachen Recht. Sie besaßen auch das Land, das von Leibeigenen bearbeitet wurde. Die Landzuteilungen an die Leibeigenen und deren Arbeitsverpflichtungen auf dem Land des Grundherren und deren sonstige Leistungspflichten waren traditionell geregelt. Die Güter waren weitgehend autark. Es gab wenig (aber etwas) Handel zwischen ihnen oder mit den (damals noch wenigen und kleinen) Städten.

Die Städte lebten vom Handwerk und Handel, aber Kosten-Nutzen-Kalküle oder Angebot und Nachfrage bestimmten auch dort noch nicht den Preis. Zünfte und Gilden legten sog. gerechte Preise fest. Abweichungen davon galten als unmoralisch. Wie auf dem Lande lag auch in den Städten politische und wirtschaftliche Macht im Mittelalter in denselben Händen.

Die Zersplitterung politischer Macht hat im europäischen Staatensystem zur Begrenzung politischer Macht geführt. Dieselbe politische Zersplitterung hat auch zu ersten Ansätzen der Begrenzung wirtschaftlicher Macht geführt. Rosenberg und Birdzell (1986: 60) schreiben zur Fusion politischer und wirtschaftlicher Macht in den Städten und zur gleichzeitigen Machtbeschränkung durch Dezentralisierung:

"Politically, they tended to be dominated by merchant families to whom trading rights and monopolies were the natural rewards of political power. But these trading monopolies were opposed by rival merchants who were intended to be excluded from the trade and who were supported in many cases by the influence and power of their own rival city-states. Unlike China and the ancient empires, the Europe of the late medieval city-states and the early monarchies came to the age of discovery without a central authority strong enough to check the determination of its merchants to gain access to profitable trading opportunities, even though some satrap or other had forbidden such access or claimed it as a private reserve. The central authorities which eventually emerged did not take the form of a single monolithic empire, but of a group of nation-states which continued among themselves the early city-state competition for trade."

Mit anderen Worten: Die politische Zersplitterung hat trotz der ursprünglichen Fusion politischer und wirtschaftlicher Macht ${ }^{6}$ innerhalb der politischen Einheiten zur Begrenzung der wirtschaftlichen Macht beigetragen - zunächst vor allem auf externen Märkten, wo politische und traditionelle Tauschbedingungen zuerst von Angebot und Nachfrage abgelöst wurden. Im Handel über politische Grenzen hinweg, mehr noch zwischen Gebieten mit unterschiedlichen Traditionen, konnte niemand politische und traditionelle Preise durchsetzen, konnten sich Marktpreise nach Angebot und Nachfrage zuerst entfalten. Die Kleinheit vieler europäischer politischer Gebilde, damit zusammenhängend deren Abhängigkeit von einem gewissen Außenhandel und natürlich der stark mit Schmuggel und Freibeuterei durchsetzte Überseehandel haben zur Befreiung europäischer Wirtschaften von traditionellen und politischen Einflüssen beigetragen. Über die größere Offenheit der Wirtschaften europäischer politischer Einheiten wurde die wirtschaftliche Macht innerhalb dieser Einheiten geschwächt und wurden ,freie' Preise und damit die Voraussetzungen einer effizienten Ressourcenallokation geschaffen. Im Laufe der Zeit haben die freien Preise im Außenverkehr dann auch die Preisgestaltung auf den Binnenmärkten beeinflußt.

\footnotetext{
${ }^{6}$ Nach Andreski (1984: 142), der diese These auf Max Weber zurückführt, ist die Trennung politischer und wirtschaftlicher Macht bedeutsamer als die Frage, wer die politische Macht innehat. Politische Macht der Kaufleute oder ,der Wirtschaft' hat ebenfalls negative Konsequenzen, denn: "new forms of production and trade (i. e. industrially oriented capitalism) develop only where the business class is too strong to be fettered and exploited but not strong enough to accumulate wealth by extracting it from others, and where, in consequence, production and trade offer to the members of this class the most promising road to satisfactory livelihood or enrichment."
} 
Wo die Märkte größer als die politischen Einheiten sind, wo restriktive Praktiken - wie Monopole und Kartelle, Zünfte und Gilden - nur innerhalb der politischen Einheiten organisiert sind, da muß auch die wirtschaftliche Macht relativ zersplittert sein, da muß ein gewisses $\mathrm{MaB}$ an Wettbewerb herrschen.

In der Dezentralisierung politischer und wirtschaftlicher Macht, nicht zuletzt in der Dezentralisierung von wirtschaftlichen und Innovationsentscheidungen kann man mit Rosenberg und Birdzell (1986: 33) eine wesentliche Quelle des Fortschritts in Europa sehen: "... the underlying source of the West's ability to attract the lightning of economic revolutions was a unique use of experiment and technology and organization to harness resources to the satisfaction of human wants. The key elements of the system were the wide diffusion of the authority and resources necessary to experiment; an absence of more than rudimentary political and religious restrictions on experiment; and incentives which combined ample rewards for success, defined as the widespread economic use of the results of experiment, with a risk of severe penalties for failing to experiment."

Die Zersplitterung politischer und wirtschaftlicher Macht und der daraus resultierende Wettbewerb haben sowohl die Notwendigkeit als auch die Möglichkeit des Fortschritts bedingt, weil viele experimentieren durften und mußten. Das ist ein ganz grundsätzlicher Punkt, auf den vor allem von Hayek (1971) immer wieder hingewiesen hat.

Fast alle Menschen wissen, wie man dieses oder jenes herstellt, wie man dieses oder jenes besser macht als andere. Dieses Wissen im weitesten Sinne fruchtbar zu machen, erlaubt nur eine dezentrale, ,spontane' oder marktwirtschaftliche Ordnung, wo jeder den Anreiz hat, seine Kenntnisse gegen Gegenleistung anderen zur Verfügung zu stellen, wo knappe (nachgefragte) Talente nicht vergeudet werden. Keine Zentralinstanz oder Behörde kann die vielen Wissensfünkchen in Tausenden oder Millionen von Köpfen zusammenfassen, koordinieren, optimal einsetzen. Natürlich wird auch in einer freien Marktwirtschaft nicht jeder seine Talente optimal nützen, aber weil er den Schaden trägt, wenn er es nicht tut, werden die meisten Leute sich zumindest darum bemühen. Die Begrenzung politischer Macht, die Trennung wirtschaftlicher und politischer Macht, die Verteilung wirtschaftlicher Entscheidungen auf eine Vielzahl möglichst konkurrierender Unternehmen ermöglicht und erzwingt Leistung, Innovation und wirt- schaftlichen Fortschritt. Man sollte in diesem $\mathrm{Zu}$ sammenhang nicht übersehen, daß die positivén Auswirkungen der Freiheit an verantwortliche Eigenentscheidungen und Konkurrenz (und damit an das Risiko des Bankrotts!) gebunden sind, daß kein Grund zur Annahme positiver Auswirkungen ,demokratischer Mitbestimmung besteht. Am deutlichsten ist das bei Innovationen. Nach Rosenberg and Birdzell (1986: 310) gilt: "a society which delayed innovations by the amount required to reach a political consensus would fall further and further behind a society which did not ... It implies the substantive criterion that the benefits of innovation are sufficiently understood and predictable, that they can be persuasively verbalized in advance of its adoption - that is, that everything is too clear to need the test of experiment."

Dieser von Eric Jones (1981) und einigen anderen übernommene Erklärungsansatz stellt die Dezentralisierung politischer und wirtschaftlicher Macht im internationalen System rivalisierender Staaten, sichere Eigentums- und Verfügungsrechte, individuelle Freiheit und Konkurrenz in den Mittelpunkt der Erklärung des europäischen ,Wirtschaftswunders', das ja schon vor der Industrialisierung begonnen hat. ${ }^{7}$ Damit stellt sich die Frage nach der Tragweite und den Grenzen des Erklärungsansatzes bzw. die Frage, wo er ergänzungsbedürftig ist.

Jones (1981) hatte sich die Aufgabe gestellt zu erklären, warum Westeuropa und nicht etwa China, Indien oder ein islamisches bzw. osmanisches Reich die weltwirtschaftliche Führung übernommen haben. Die Frage, warum sich zunächst die Niederlande und England und erst später die anderen europäischen Länder kapitalistisch entwickelt haben, tritt bei ihm in den Hintergrund. Soweit läßt der Erklärungsansatz Fragen offen. Man kann darüber hinaus fragen, ob die Last eines geeinten Reiches und damit einhergehend unsichere Eigentums-, Freiheits- und Verfügungsrechte in China, Indien und im Vorderen Orient gleich bedeutsam waren. Was die nur schwach entwickelten individuellen Eigentums-, Freiheits- und Verfügungsrechte angeht, kann man das $m$. E. bejahen. Aber nach Jones (1981) und z. T. den anderen zitierten Autoren hängt dieses Defizit ja eng mit der politischen Einheit eines Kulturkreises zusammen, kann

\footnotetext{
${ }^{7}$ Nach Chirot (1986: 59) hatten England, die Niederlande und die Vereinigten Staaten schon ca. 1800 ein Sozialprodukt pro Kopf, das über dem vieler Staaten Anfang der 1980er Jahre liegt, in denen über die Hälfte der zeitgenössischen Menschheit lebt.
} 
dieses Defizit nur in einem System rivalisierender Staaten überwunden werden.

In China ist die politische Einheit des Landes seit ca. 2200 Jahren die Norm, gelten Desintegrationserscheinungen als pathologisch und vorübergehend. Es hat zwar Unruhen und Bürgerkriege, aber kein chinesisches Staatensystem gegeben, wo sich wie in Europa annähernd gleichwertige Staaten gegenüberstanden. Auch Hall (1986: 34), an dem ich mich im folgenden orientieren will, kommt deshalb bei China zum selben Schluß wie Jones (1981): "economic dynamism was curtailed by the imperial form."

Während China über lange Zeiträume ein einziges Reich war, wurde die indische Zivilisation nur dreimal politisch geeint - unter den Mauryas im dritten Jahrhundert vor unserer Zeitrechnung, später von den islamischen Moguln und zuletzt von den britischen Kolonialherren. Zwar kannte auch Indien keine sicheren Freiheits-, Verfügungs- und Eigentumsrechte, aber die politische Einheit des Landes war zu brüchig, um als Erklärung dienen zu können. Mit Hall (1986) kann man schon eher darauf verweisen, daß in Indien gerade die Instabilität der politischen Systeme einen kurzfristigen Kalkulationshorizont der Herrscher bedingt hat. Dieser kurzfristige Horizont hat die Überwindung der an Beute und Untertanenausplünderung orientierten Herrschaft und die Entstehung sicherer Verfügungs- und Eigentumsrechte behindert. In Europa dagegen waren viele der miteinander rivalisierenden politischen Einheiten relativ stabil über die Zeit. Ein stabiles internationales System und instabile politische Einheiten wechselnder Größe implizieren zwar beide fast permanente bewaffnete Konflikte, haben aber unterschiedliche Auswirkungen auf die Ausbildung von Eigentums-, Freiheits- und Verfügungsrechten.

Warum sich Indien nicht wie Westeuropa entwikkelt hat, kann m. E. auch nicht ohne Rückgriff auf den Hinduismus und das Kastensystem erklärt werden (vgl. Hall 1986, Weber 1978, Weede 1985a). Mit seinen detaillierten Arbeits- und Verbrauchsvorschriften ist das indische Kastenwesen innovationsfeindlich. Wer neue Arbeitstechniken verwendet, muß sich im Hinduismus fragen, ob diese noch mit seiner Kastenpflicht kompatibel sind, ob die Innovation nicht sein Wiedergeburtsschicksal negativ beeinflußt. Weil Vernachlässigung der Kastenpflichten magisches Unheil über die ganze Kaste bringen könnte, hat auch die Kaste als solche ein Interesse daran, soteriologisch immer fragwürdige Innovationen einzelner Mit- glieder zu unterbinden. Sorge um das Schicksal im nächsten Leben und sozialer Druck in diesem Leben zusammen sind dabei eine mächtige Innovationsbremse. Individueller Aufstieg in diesem Leben ist ohnehin nicht vorgesehen. Man könnte diese Anreizstruktur nicht nur als unkapitalistisch, sondern geradezu als anti-ökonomisch bezeichnen.

Im islamisch-osmanischen Raum treten teilweise die Probleme Chinas, teilweise einige Probleme Indiens auf. Das osmanische Reich hat jahrhundertelang große Teile der islamischen Weit beherrscht, hat nur an seiner Peripherie mit anderen und meist schwächeren und instabilen politischen Systemen Kontakt gehabt. Die Last des Imperiums bzw. das Fehlen eines Systems konkurrierender Staaten können also erklären, warum der osmanische Staat eine Plünderungsmaschine war, warum sich keine sicheren Eigentums- und Verfügungsrechte entwickelten, warum die Innovationsanreize so gering waren. Außerhalb des osmanischen Reiches ist die islamische Welt jahrhundertelang eher durch politische Instabilität gekennzeichnet gewesen (vgl. Hall 1986), die - wie am indischen Beispiel schon besprochen - ebenfalls keine sicheren Eigentums- und Verfügungsrechte begünstigt. Offen bleibt $\mathrm{m}$. E. nur noch die Frage, warum das osmanische Reich in Anbetracht des engen Kontaktes zum europäischen Staatensystems nicht etwas mehr von dessen Dynamik abbekommen hat jedenfalls dann wenn man die europäische Dynamik so ganz ohne religionssoziologische Überlegungen etwa im Sinne Webers (1972) erklären will. Ähnlich wie bei Collins (1980) und bei Weber (1981) selbst in seiner Wirtschaftsgeschichte wird hier die protestantische Ethik bzw. die Suche nach Heilsgewißheit durch Geschäftserfolg zu einem eher sekundären Element bei der Erklärung des europäischen Sonderweges. ${ }^{8}$

${ }^{8}$ Um Mißverständnisse bei denen zu vermeiden, die mit Webers (1972) ,Protestantischer Ethik' nicht vertraut sind, möchte ich nur unterstreichen, daß Geschäftserfolg nicht Mittel sein kann, um zum Heil zu gelangen, sondern nur Mittel, um die Angst um das eigene Heil zu bewältigen. - Während ich in meiner Erklärungsskizze des westlichen Sonderweges auf die ,Protestantische Ethik' kaum zurückgegriffen habe, habe ich mir Webers religionssoziologische Thesen zumHinduismus in stärkerem Ausmaß zu eigen gemacht. Ähnlich wie Hall (1986) halte ich die politischen Implikationen des Hinduismus und daraus hervorgegangener Lehren aber für ebenso bedeutsam wie dessen Wirtschaftsethik. 
Bei einem Erklärungsversuch für den westlichen Sonderweg, der so stark wie meiner auf die positiven Folgen der politisch-militärischen Rivalitäten in Europa abstellt, stellen die Vereinigten Staaten von Amerika und deren kapitalistische Entwicklung ein Problem dar. Denn Amerika hat sich ja vor dem ersten Weltkrieg gut ein Jahrhundert aus den Konflikten der europäischen Staaten herausgehalten und in seiner eigenen Hemisphäre nie gleichwertige Gegner gehabt. Dennoch kann ich die erfolgreiche kapitalistische Entwicklung Amerikas nicht als entscheidenden Einwand gegen meine Erklärungsskizze akzeptieren. Denn erstens haben sich kapitalistische Wirtschaftsformen zuerst in Europa, vor allem in Holland und England, herausgebildet und sind erst später, nicht zuletzt von europäischen Einwanderern nach Amerika gebracht worden. Auch die industrielle Revolution als Beschleunigung der kapitalistischen Entwicklung hat sich in England, nicht etwa in Amerika, zuerst ereignet. Denn zweitens ist die zwischenstaatliche Rivalität in meiner, vor allem auf Jones (1981) aufbauender Erklärungsskizze ja nur eine recht indirekte Determinante der kapitalistischen Wirtschaftsentwicklung.

Zwischen internationaler Rivalität und Wirtschaftsentwicklung intervenieren vor allem die Sicherheit der Eigentumsrechte, eine kalkulierbare Rechtsordnung, dezentralisierte Entscheidungen und Produktionsanreize. Dazu können auch andere hetrschaftsbeschränkende Gegebenheiten als internationale Rivalitäten beitragen. In Amerika sind das einmal die im 19. Jahrhundert nach Westen offene Grenze, zum anderen die zwar (vom Bürgerkrieg abgesehen) nicht-militärischen, aber dennoch vorhandenen Rivalitäten zwischen den amerikanischen Einzelstaaten, die ja bis ins 20 . Jahrhundert hinein ihre staatlichen Rechte eifersüchtig bewahrt haben. Gerade weil Bürger, Talent und bewegliches Kapital leicht von einem Gliedstaat in einen anderen übersiedeln konnten, hat das politische Übergriffe in die Wirtschaft drastisch beschränkt.

Mit Rosenberg and Birdzell (1986: 138) kann man deshalb durchaus eine Parallele zwischen dem europäischen und dem amerikanischen Staatenpluralismus in der Vergangenheit sehen: "It may be that a prerequisite to sustained economic growth is an economy trading across a geographical area divided among a number of rival states, each too small to dream of imperial wars and too fearful of the economic competition of other states to impose massive exactions on its own sphere. The United
States had a federal system in the nineteenth and early twentieth centuries in which political intervention by the national government was narrowly restricted by political tradition and constitutional interpretation, while political intervention by the state governments was restricted by the fear of economic competition from other states. Whether the constitutional reconstruction of the United States as a classic empire is compatible with indefinitely sustained growth is, of course, a topical and controversial question." Danach kann nur die Beschränkung politischer Macht, ob durch ein Mächtegleichgewicht, eine Verfassung (die ja ihrerseits die Machtverhältnisse unter den Gründerstaaten der Union widerspiegelt) bzw. eine Mischung beider Elemente, der Wirtschaft den nötigen Wachstumsspielraum verschaffen. ${ }^{9}$

${ }^{9}$ Mit Berger (1986) bin ich der Meinung, daß eine umfassende Theorie der kapitalistischen Entwicklung, nicht nur den Sonderweg des Westens, sondern auch die erfolgreiche Entwicklung Japans, Südkoreas, Taiwans, Hongkongs und Singapurs erklären muß. Das kann im Rahmen dieses Aufsatzes nicht versucht werden. Aber ich möchte anmerken, daß zwischenstaatliche Rivalität und sicherheitspolitische Gesichtspunkte bei der japanischen, südkoreanischen und taiwanesischen Entwicklung eine wichtige Rolle gespielt haben, $\mathrm{da} B$ die kleineren ostasiatischen Staaten sehr stark exportorientiert sind, daß Hongkong sich bei weitestgehendem Freihandel entwickelt hat. Das sind Tatsachen, die sich recht gut in meinen Erklärungsansatz einfügen lassen. Problematischer ist eine andere Tatsache, nämlich daß der Staat in Japan, Südkorea und Taiwan recht stark in die wirtschaftliche Entwicklung eingegriffen hat. Gerade wegen der Dominanz sicherheitspolitischer Uberlegungen auch in der Wirtschaftspolitik Japans (vor 1945), Südkoreas und Taiwans könnten die Staatseingriffe in diesen Fällen weniger als anderswo zu Preisverzerrungen und damit zur Beeinträchtigung der allokativen Effizienz geführt haben. Leider gibt die World Bank (1983: 62) nur für Südkorea, nicht für Japan oder Taiwan Preisverzerrungsindices an. In Südkorea sind die Preisverzerrungen danach gering - vor allem auf dem Arbeitsmarkt. Die negativen Erfahrungen, die Singapur in den frühen 80er Jahren mit dem Versuch machte, das Lohnniveau anzuheben, deuten an, daß staatliche Zurückhaltung gerade auf dem Arbeitsmarkt wichtig ist. 


\section{Wirtschaft, Wissenschaft und Demokratie}

Der europäische Sonderweg erschöpft sich nicht in der Erfindung des Kapitalismus ${ }^{10}$ und in einem Wirtschaftswachstum, das erstmals in der Menschheitsgeschichte die Überwindung von Massenarmut und Massenelend gestattet hat. In Europa und seinen Tochtergesellschaften sind auch die moderne Wissenschaft und die Demokratie entstanden. In meiner Erklärungsskizze oben habe ich die europäische Wirtschaftsentwicklung wesentlich auf die Beschränkung der Herrschaft durch zwischenstaatliche Rivalität und innenpolitische Pattsituationen und die dadurch ermöglichte Entwicklung des Rechts, d.h. vor allem der Eigentumsrechte, zurückgeführt. Offensichtlich ist die weitgehende Einschränkung herrschaftlicher Willkür nicht nur Voraussetzung für eine autonome und dynamische Wirtschaftsentwicklung, sondern auch für eine den Erkenntnisfortschritt vorantreibende Wissenschaftsentwicklung und für die Entstehung der Demokratie. Der europäische Sonderweg besteht darin, in Wirtschaft, Wissenschaft und Politik die Voraussetzungen für eine rationale Praxis geschaffen zu haben, die in Anbetracht der Fehlbarkeit menschlicher Entscheidungen vor allem Raum zur kritischen Prüfung und zur Korrektur von Fehlentscheidungen lassen muß (Albert 1978, Radnitzky 1987a, 1987b). In dieser Hinsicht haben Preis- und Qualitätskonkurrenz auf dem Markt, theoretische Auseinandersetzungen und möglichst exakte - im Idealfalle experimentelle - Überprüfung und Parteienpluralismus einschließlich innerparteilicher Konflikte letztlich dieselbe Funktion.

Die Korrigierbarkeit von Fehlentscheidungen setzt voraus, daB niemand die Macht hat, einmal getroffene Entscheidungen gegen Kritik zu immunisieren. Ein wichtiger Mechanismus zur Sicherung der Korrigierbarkeit von Entscheidungen und zur Minimierung von Macht und Herrschaft besteht in der möglichst weitgehenden Autonomie verschiedener Lebensbereiche, wie Wirtschaft, Wissen-

${ }^{10}$ Wenn man Kapitalismus mit Berger (1986: 19) durch "production for the market by enterprising individuals or combines with the purpose of making a profit" definiert, dann sind Kapitalismus und Marktwirtschaft Synonyme. Man kann Kapitalismus natürlich auch durch Privatbesitz an Produktionskapital definieren. Weil ich mit Berger (1986: 190) der Auffassung bin, $\mathrm{da} B$ es ohne Privatbesitz an Produktionskapital keine funktionierende Marktwirtschaft geben kann, verwende ich Kapitalismus und Marktwirtschaft als Synonyme. schaft und Politik von einander. Wo die Politik die Wirtschaft dominiert, verkümmern die Arbeitsund Innovationsanreize für die produktiv Tätigen. Das war in den außereuropäischen Agrargesellschaften der Fall und hatte die von Albert (1986: 15) mit folgenden Worten beschriebenen Konsequenzen: „Die normale Staatsidee der vorindustriellen Epoche war, so könnte man sagen, die der Eroberung und der Ausbeutung. Die Hochkulturen waren weitgehend auf Zwangsarbeit gegründet und durch eine endemische Rechtlosigkeit der unterdrückten Bevölkerung gekennzeichnet ... Was die Situation der Massen in diesen Kulturen angeht, so sind die vorherrschenden Lebensbedingungen stets die des größten Elends gewesen."

Auch in den sozialistischen Planwirtschaften von heute dominiert die Politik die Wirtschaft mit tendenziell ähnlichen Konsequenzen. Die Rechtlosigkeit der Bevölkerung wird durch Ausreiseverbote, Kriminalisierung von Republikflucht, Mauern und Todesstreifen sichtbar gemacht. Materielles Massenelend haben die osteuropäischen (nicht aber die ostasiatischen) Planwirtschaften inzwischen zwar überwunden, aber ein Einholen oder Überholen der westlichen Marktwirtschaften erscheint auf absehbare Zeit ausgeschlossen (Berger 1986, Chirot 1986). Das gilt sogar dann, wenn man - wie Pryor (1985) - gleich schnelles Wirtschaftswachstum in kapitalistischen und sozialistischen Volkswirtschaften unterstellt. Jedenfalls im zivilen Bereich sind die kapitalistischen Gesellschaften wesentlich innovativer und produktiver als die sozialistische Alternative, so da $ß$ man die Frage aufwerfen - und m. E. bejahen - kann, ob nicht die Erfolge nachholender Industrialisierung im sozialistischen Osten wesentlich auf der Übernahme westlicher Produktionstechniken beruhen. $\mathrm{DaB}$ die Freiheit im Westen auch dem Osten zugute kommen kann, wird etwa von folgender These von Hayeks (1971: 42) impliziert: „Es kann kein Zweifel bestehen, daß im Laufe der Geschichte unfreie Mehrheiten Gewinn aus der Freiheit einer Minderheit gezogen haben und $d a ß$ heute unfreie Gesellschaften Gewinn aus Dingen ziehen, die sie von freien Gesellschaften erhalten und lernen."

Umgekehrt ist eine Beherrschung der Politik durch die Wirtschaft - sei es durch Großunternehmen oder Gewerkschaften - genauso wenig wünschenswert wie der Durchgriff der Politik auf die Wirtschaft. Denn politischer Einfluß der Wirtschaft, d.h. von Unternehmen und Verbänden, lädt ja dazu ein, mit politischen Mitteln dem Wettbewerb zu entkommen. Das Interesse eines jeden Teilneh- 
mers am Wirtschaftsgeschehen liegt ja darin, vom Wettbewerb unter den anderen zu profitieren, selbst aber dem Leistungszwang des Wettbewerbs zu entkommen. Unternehmen, zumindest teilweise einschließlich ihrer Beschäftigten, haben zwar ein Interesse daran, daß auf den Märkten, wo sie ihre Inputs beziehen, Wettbewerb herrscht, würden aber gern auf ihrem Output-Markt Wettbewerbsbeschränkungen durchsetzen, etwa durch möglichst hohe Marktzugangsschranken für ausländische Produzenten. Nur eine möglichst weitgehende Autonomie von Wirtschaft und Politik kann gleichzeitig Ausbeutung und restriktive Praktiken verhindern, kann gleichzeitig Arbeits- und Innovationsanreize und damit eine fortlaufende Verbesserung der materiellen Lebensumstände der Menschen garantieren.

Wie Wirtschaft und Politik von einander relativ unabhängig sein sollten, so sollte auch die Wissenschaft ein gegenüber beiden Bereichen autonomer Bereich sein. Nach Kuhn (1976: 175) bedeutet die soziale und institutionelle Absicherung der wissenschdftlichen Unabhängigkeit vor allem auch, daß „die kreative Arbeit des einzelnen ... ausschließlich an andere Mitglieder der Gruppe (der Wissenschaftler, E. W.) gerichtet ist und von diesen bewertet wird." Wissenschaftliche Autonomie impliziert also, daß Wissenschaftler für einander arbeiten und einander kritisieren. Gerade weil Eingriffe politischer und religiöser Instanzen historisch immer wieder die Gedankenfreiheit bedroht haben und auch künftig bedrohen könnten, muß die „Zähmung der Herrschaft“ (Albert 1986) ergänzt werden durch soziale Normen, die verhindern, daß Wissenschaftler selbst die Politik in Auseinandersetzungen um Erklärungen und Problemlösungen instrumentalisieren. Mit den Worten von Kuhn (1976: 179/180): „Eine der stärksten, wenn auch noch ungeschriebenen Regeln des wissenschaftlichen Lebens ist das Verbot von Appellen an Staatsoberhäupter oder die ganze Bevölkerung in Angelegenheiten der Wissenschaft." Bedeutsam an dieser Aussage ist, daß die Autonomie der Wissenschaft der entscheidende Wert ist und Übergriffe demokratisch legitimierter Instanzen in die Wissenschaft genauso problematisch sind wie solche nicht-demokratischer politischer Instanzen. Für den Erkenntnisfortschritt kommt es auf die Abwehrbarkeit aller sachfremden Einflüsse an.

Dazu kann auch die vermeintliche soziale Dringlichkeit mancher Probleme gehören. Kuhn (1976: 175) hebt hervor, „daß die Absonderung der wissenschaftlichen Gemeinschaft von der Gesellschaft es dem einzelnen Wissenschaftler erlaubt, seine Aufmerksamkeit auf Probleme zu konzentrieren, von denen er begründet annehmen darf, daß er sie lösen kann." Die Autonomie der Wissenschaft gegenüber den Mächtigen in den Bereichen der Politik oder auch der Wirtschaft ist dabei als institutionelle Voraussetzung des Erkenntnisfortschritts gedacht - und damit letzten Endes auch im Interesse der Gesellschaft.

Autonomie der Wissenschaft wie Autonomie der Wirtschaft hat dabei die Funktion, das Ausmaß an Selbstbestimmung in den betroffenen Lebensbereichen möglichst groß zu halten, für das bloße Mitbestimmung weder im einen noch im anderen Bereich ein Ersatz sein kann. Weil die Beschränkung der Herrschaft eine notwendige Voraussetzung von Erkenntnisfortschritt ist, weil auch die Demokratie immer noch eine Form der Herrschaft und nicht etwa deren Begrenzung oder gar Überwindung ist (Albert 1978: 55/56, von Hayek 1971, Radnitzky 1987a, 1987b), bleibt die wissenschaftliche Autonomie in der Demokratie genauso wie in anderen Herrschaftsformen ein Desideratum.

Obwohl die „Befreiung vom Interesse an unmittelbarer praktischer Verwertung“ (Albert 1986: 36), einschließlich unmittelbarer wirtschaftlicher Verwertung, zum Erkenntnisfortschritt beiträgt, hat die Wissenschaft spätestens seit dem Ende des 19. Jahrhunderts wesentlich zum Wirtschaftswachstum beigetragen (Chirot 1986: 208, Kuznets 1976, Rosenberg and Birdzell 1986). Die Autonomie von Wirtschaft und Wissenschaft gegenüber der Politik hat nicht nur in der, Zähmung der Herrschaft (Albert 1986) eine gemeinsame Wurzel, sondern sie hat auch einander verstärkende Effekte. Erkenntnisfortschritte erlauben neue Produktionsverfahren. Zunehmender Wohlstand erlaubt die Freisetzung von immer mehr Menschen für wissenschaftliche Tätigkeit.

Bisher habe ich immer wieder auf die positiven Folgen der Begrenzung politischer Herrschaft verwiesen, aber das Thema Demokratie nur am Rande behandelt. Offensichtlich setzt Demokratie starke Beschränkung von Herrschaft, auch der Mehrheitsherrschaft, voraus, wenn sie mehr als eine Farce sein soll. Die Demokratie ist nicht mit der Aufhebung von Meinungs-, Rede-, Presse- und Vereinigungsfreiheit kompatibel. Die jeweils Herrschenden dürfen die nächste Wahl auch nicht beliebig lang hinausschieben können. Das sind so offensichtliche Merkmale demokratischer Regime, daß sie oft in Definitionen der Demokratie aufgenommen oder stillschweigend vorausgesetzt wer- 
den. Man kann aber darüber hinaus in der Existenz einer gegenüber der Politik autonomen Wirtschaft, d. h. im Kapitalismus oder in der Marktwirtschaft, eine notwendige Voraussetzung für die Existenz von Demokratien sehen (von Hayek 1971, 1976; Berger 1986: 74-81). Tatsache ist jedenfalls, daß bisher nur kapitalistische Gesellschaften (wenn auch nicht alle kapitalistischen Gesellschaften) Demokratien mit vom Volk abwählbaren Regierungen sind.

Die beobachtbare, wenn auch nicht perfekte Affinität von Kapitalismus und Demokratie beruht einmal auf der von beiden vorausgesetzten Einschränkung von Herrschaft. Sie beruht darauf, daß der Markt die Koordination menschlicher Tätigkeiten ohne politische Vorgaben gestattet und damit die Politik wesentlich entlastet (Friedman 1976: 36). Von besonderer Bedeutung ist dabei die Entlastung der Politik von Verteilungsentscheidungen, weil sich das demokratische Mehrheitsprinzip gerade zu Verteilungsentscheidungen besonders wenig eignet.

Das hat Usher (1981) in einem Gedankenexperiment illustriert. Man denke sich eine Gesellschaft mit nur 15 erwachsenen Mitgliedern, wo die Demokratie noch direkt sein kann und Repräsentations- und daraus resultierende Oligarchieprobleme entfallen. Das Volkseinkommen soll außerdem wie Manna vom Himmel fallen, damit auch das Problem der Arbeitsanreize und -organisation entfällt. Es geht nur darum, 300000 Dollar unter 15 Personen zu verteilen. Wenn diese Personen eigennützig sind, dann kann das demokratische Mehrheitsprinzip diese Aufgabe nicht stabil lösen.

Natürlich kann man die 300000 durch 15 dividieren und jedem 20000 Dollar zuweisen. Aber eine eigennützige Mehrheit von 8 Bürgern kann auch beschließen, 300000 durch $8 \mathrm{zu}$ dividieren, jedem Koalitionsmitglied 37500 Dollar zu geben und den anderen gar nichts zu überlassen. Danach lohnt es sich für die Verlierer der Abstimmung, einem Koalitionsmitglied mehr als 37500 Dollar zu bieten und mit ihm eine neue Mehrheit zu bilden, die sieben der acht ursprünglichen Koalitionsmitglieder enteignet. Aber dieses Ergebnis wird genauso wenig stabil wie das der ersten Abstimmung sein. Eine Stabilisierung irgendeines Verteilungsergebnisses ist nur durch Suspendierung des demokratischen Mehrheitsprinzips zu irgendeinem Zeitpunkt möglich.

Indem der Markt der damit überforderten Demokratie die Verteilungsentscheidungen abnimmt, gleichzeitig auch noch die Probleme der Arbeitsan- reize und -organisation löst, gleichzeitig auch noch durch seine Unabhängigkeit von politischer Macht zu deren Begrenzung beiträgt, verbessert er die Überlebenschancen der Demokratie.

\section{Abschließende Überlegungen}

Meine Erklärungsskizze für den Sonderweg des Westens ist unter mehreren Gesichtspunkten offen. Erstens handelt es sich nur um eine Erklärungsskizze und nicht um eine Theorie, die den Desiderata der (von mir akzeptierten) Wissenschaftslehre oder Methodologie genügt und hinreichend strengen Tests unterzogen worden ist oder auch nur unterzogen werden kann.

Zweitens ist nicht nur die Erklärung des westlichen Sonderweges offen, sondern auch dessen Fortsetzung. Im Mittelpunkt meiner Erklärungsskizze steht die Kausalkette Konflikt-Freiheit-Fortschritt, wobei sich der Konflikt auf internationale Rivalitäten ebenso wie auf innerstaatliche Interessengegensätze bezieht, wobei die Freiheit den Entscheidungsspielraum von Individuen zur Selbstbestimmung meint, wobei die Freiheit wesentlich auch von der relativen Autonomie der Lebensbereiche Wirtschaft, Wissenschaft und Politik voneinander abhängt, wobei der Fortschritt im Sinne von Hayeks (1971; vgl. auch Radnitzky 1987a, 1987b) als Folge der optimalen Nutzung des immer dezentralisierten Wissens in Millionen Köpfen verstanden wird. Für die Vergangenheit sollte man m. E. den Sonderweg des Westens als eine Erfolgsgeschichte auffassen. Ich neige auch dazu, die wichtigsten westlichen Errungenschaften - die relative Autonomie von Wirtschaft, Wissenschaft und Politik; die Freiheit des Individuums; die freie Marktwirtschaft - den Entwicklungsländern zur Nachahmung zu empfehlen (vgl. Weede 1985a, 1985b).

Aber der bisherige Erfolg und der exemplarische Charakter des westlichen Sonderweges reichen natürlich nicht aus, um die Überlegenheit oder auch nur Überlebensfähigkeit des Westens und seiner Errungenschaften zu prognostizieren. In Bezug auf die Zukunft des Westens lassen sich die Fragezeichen gleich mehrfach rechtfertigen: Die Freiheit der Märkte und des Wettbewerbs wird durch Verteilungskoalitionen, restriktive Praktiken und den Wohlfahrtsstaat beschnitten und die Wachstumsraten der westlichen Industrieländer sinken deshalb (Bernholz 1986, Olson 1985, Weede 1986a, 1986b). Den freiheitlichen Demokratien fällt die militärische Rivalität mit anderen politischen Systemen schwer (Bernholz 1985: 98-99, 159; 
Kammler 1986, Weede 1986a, 1986c). Es ist sogar denkbar, daß kapitalistische Gesellschaften auf soziale (Hirsch 1980) oder natürliche Wachstumsgrenzen stoßen. Aber die Behandlung dieser Fragen verdient separate und zusätzliche Anstrengungen.

\section{Literatur}

Albert, Hans, 1978: Traktat über rationale Praxis, Tübingen: Mohr.

Albert, Hans, 1986: Freiheit und Ordnung, Tübingen: Mohr.

Andreski, Stanislav, 1968a: Military Organization and Society, 2nd ed., Stanford: University Press.

Andreski, Stanislav, 1968b: The African Predicament, New York: Atherton Press.

Andreski, Stanislav, 1984: Max Weber's insights and errors, London: Routledge and Kegan Paul.

Berger, Peter L., 1986: The Capitalist Revolution. Fifty Propositions About Prosperity, Equality and Liberty, New York: Basic Books.

Bernholz, Peter, 1985: The International Game of Power, Berlin-New York-Amsterdam: Mouton.

Bernhoiz, Peter, 1986: Growth of Government, Economic Growth and Individual Freedom. Journal of Institutional and Theoretical Economics (Zeitschrift für die gesamte Staatswissenschaft) 142, 661-683.

Bornschier, Volker (ed.), 1980: Multinationale Konzerne, Wirtschaftspolitik und nationale Entwicklung im Weltsystem. Frankfurt/Main: Campus.

Brunner, Karl, 1985: The Poverty of Nations. Cato Journal 5(1): 37-49.

Chirot, Daniel, 1986: Social Change in the Modern Era, San Diego: Harcourt, Brace, Jovanovich.

Collins, Randall, 1980: Weber's Last Theory of Capitalism: A Systematization. American Sociological Review 45, 925-942.

Dixon, William J. and Moon, Bruce E., 1986: The Military Burden and Basic Human Needs. Journal of Conflict Resolution 30, 660-684.

Durkheim, Emile, 1977: Über die Teilung der sozialen Arbeit, Frankfurt/Main: Suhrkamp.

Ekelund, Robert B. and Tollison, Robert D., 1981: Mercantilism as a Rent-Seeking Society. Economic Regulation in Historical Perspective, College Station: Texas $A$ and $M$ University Press.

Emmanuel, Arghiri, 1972: Unequal Exchange: A Study of the Imperialism of Trade, New York: Monthly Review Press.

Frank, Andre Gunder, 1978a: Dependent Accumulation and Underdevelopment, London: Macmillan.

Frank, Andre Gunder, 1978b: World Accumulation, 1492-1789, London: Macmillan.

Friedman, Milton, 1976: Kapitalismus und Freiheit, München: DTV.

Galtung, Johan, 1972: Eine strukturelle Theorie des Imperialismus. S. 29-104 in Senghaas, Dieter (ed.): Im- perialismus und strukturelle Gewalt, Frankfurt/Main: Suhrkamp.

Garnier, Maurice A. and Hazelrigg, Lawrence E., 1977: Military Organization and Distributional Inequality. Journal of Political and Military Sociology 5, 17-33.

Hall, John A., 1986: Power and Liberties. The Causes and Consequences of the Rise of the West, Berkeley: University of California Press.

Hayek, Friedrich August von, 1971: Die Verfassung der Freiheit, Tübingen: Mohr.

Hayek, Friedrich August von, 1976: Der Weg in die Knechtschaft, München: DTV.

Hirsch, Fred, 1980: Die sozialen Grenzen des Wachstums, Reinbek bei Hamburg: Rowohlt.

Jagodzinski, Wolfgang und Weede, Erich, 1980: Weltpolitische und ökonomische Determinanten einer ungleichen Einkommensverteilung. Zeitschrift für Soziologie 9, 132-148.

Jones, Eric L., 1981: The European Miracle, Cambridge: University Press.

Kahn, Herman, 1979: World Economic Development, London: Croom and Helm.

Kammler, Hans, 1986: Versagen der Demokratien? Zeitschrift für Politik 33, 235-253.

Kriesberg, Louis, 1979: Social Inequality, Englewood Cliffs, New Jersey: Prentice-Hall.

Kuhn, Thomas S., 1976: Die Struktur wissenschaftlicher Revolutionen, Frankfurt/Main: Suhrkamp.

Kuznets, Simon, 1976: Modern Economic Growth: Rate, Structure and Spread, New Haven, Conn.: Yale University Press (7th ed.).

McNeill, William H., 1982: The Pursuit of Power, Chicago: University Press.

North, Douglass C., 1981: Structure and Change in Economic History, New York: Norton.

North, Douglass C. and Thomas, Robert Paul, 1973: The Rise of the Western World: A New Economic History, Cambridge: University Press.

Olson, Mancur, 1985: Aufstieg und Niedergang von Nationen, Tübingen: Mohr.

Pryor, Frederic L., 1985: Growth and Fluctuations of Production in OECD and East European Countries. World Politics 37, 204-237.

Radnitzky, Gerard, 1987a: The Constitutional Protection of Liberty. S. 17-46 in Eamonn Butler and Madsen Pirie (eds.): Hayek on the fabric of human society, London: Adam Smith Institute.

Radnitzky, Gerard, 1987b: An Economic Theory of the Rise of Civilization and Its Policy Implications: Hayek's Account Generalized. Ordo 38, 47-90.

Rosenberg, Nathan and Birdzell, L. E., 1986: How the West Grew Rich, New York: Basic Books.

Ruestow, Alexander, 1950: Ortsbestimmung der Gegenwart. 1. Band, Erlenbach-Zürich: Rentsch.

Tilly, Charles, 1985: War Making and State-Making as Organized Crime. S. 169-191 in: Evans, Peter B., Rueschemeyer, Dietrich and Skocpol, Theda (eds.): Bringing the State Back In, Cambridge: University Press. 
Tullock, Gordon, 1974: The Social Dilemma. The Economics of War and Revolution, Blacksburg, Va.: University Publications.

Usher, Dan, 1981: The Economic Prerequisite to Democracy, Oxford: Basil Blackwell.

Wallerstein, Immanuel, 1974: The Modern World System: Capitalist Agriculture and the Origins of the European World Economy in the Sixteenth Century, New York: Academic Press.

Wallerstein, Immanuel, 1979: The Capitalist World-Economy. Cambridge: University Press.

Weber, Max, 1972: Gesammelte Aufsätze zur Religionssoziologie. 1. Band, Tübingen: Mohr (6. Aufl.).

Weber, Max, 1978: Gesammelte Aufsätze zur Religionssoziologie. 2. Band, Tübingen: Mohr (6. Aufl.).

Weber, Max, 1981: Wirtschaftsgeschichte, Berlin: Duncker und Humblot (4. Aufl.).

Weede, Erich, 1983: Military Participation Ratios, Human Capital Formation and Economic Growth. Journal of Political and Military Sociology 11, 11-19.

Weede, Erich, 1985a: Entwicklungsländer in der Weltgesellschaft, Opladen: Westdeutscher Verlag.
Weede, Erich, 1985b: Warum bleiben arme Leute arm? Rent-Seeking und Dependenz als Erklärungsgrundlage für die Armut in der Dritten Welt. Politische Vierteljahresschrift 26, 270-286.

Weede, Erich, 1986a: Konfliktforschung. Opladen: Westdeutscher Verlag.

Weede, Erich, 1986b: Sectoral reallocation, distributional coalitions and the welfare state as determinants of economic growth rates in industrialized democracies. European Journal of Political Research 14, 501-520.

Weede, Erich, 1986c: Nutzentheoretische Überlegungen zur (westlichen) Abschreckungspolitik. Zeitschrift für Politik 33, 254-274.

Weede, Erich and Jagodzinski, Wolfgang, 1981: National Security, Income Inequality and Economic Growth, in: Social Science and Policy Research (Seoul, S. Korea) III(3), 91-107. Wieder abgedruckt S. 269-288 in: Paelink, Jean H. P. and Vossen, P. H. (eds.), 1987: Axiomatics and Pragmatics of Conflict Analysis. London: Gower.

World Bank, 1983: World Development Report, New York: Oxford University Press. 